\title{
The Sprague-Grundy Functions of Saturations of Misère Nim
}

\author{
Yuki Irie* \\ Research Alliance Center for Mathematical Sciences \\ Tohoku University \\ Miyagi, Japan \\ yirie@tohoku.ac.jp
}

Submitted: Aug 1, 2019; Accepted: Mar 8, 2021; Published: Mar 26, 2021

(C) The author. Released under the CC BY license (International 4.0).

\begin{abstract}
We consider misère Nim as a normal-play game obtained from Nim by removing the terminal position. While explicit formulas are known for the Sprague-Grundy functions of Nim and Welter's game, no explicit formula is known for that of misère Nim. All three of these games can be considered as position restrictions of Nim. What are the differences between them? We point out that Nim and Welter's game are saturated, but misère Nim is not. Moreover, we present explicit formulas for the Sprague-Grundy functions of saturations of misère Nim, which are obtained from misère Nim by adjoining some moves.
\end{abstract}

Mathematics Subject Classifications: 91A46

\section{Introduction}

The loser in Nim is the winner in misère Nim. Nim is a two-player game played with heaps of coins. Two players alternately choose a heap and take at least one coin from it. The player who takes the last coin wins in Nim and loses in misère Nim. In general, the player who moves last wins in the normal-play convention and loses in the misèreplay convention, which has been extensively studied by using misère Sprague-Grundy functions, genera, and misère quotients (see, for example, $[4,5,8,12,13]$ ). In this paper, we will consider misère Nim as a normal-play game obtained from Nim by removing the terminal position (see Section 1.2).

Impartial games including Nim and misère Nim can be analyzed using their (normal) Sprague-Grundy functions $[7,15]$. Sprague-Grundy functions are defined recursively, and

*This work was partially supported by JSPS KAKENHI Grant Number JP20K14277. Part of the work was carried out at Chiba University. 
computing them often leads to a combinatorial explosion. However, explicit formulas are known for the Sprague-Grundy functions of some games such as Nim [7,15] and Welter's game [16], which is a position restriction of Nim. Though misère Nim is also a position restriction of Nim, no explicit formula is currently available for its SpragueGrundy function. ${ }^{1}$

What are the differences between Nim, Welter's game, and misère Nim? One of the differences is that Nim and Welter's game are 2-saturated ${ }^{2}$, but misère Nim is not. The purpose of this paper is to present an explicit formula for the Sprague-Grundy functions of 2-saturations of misère Nim, which are obtained from misère Nim by adjoining some moves. More generally, for a mixed-radix number system $\beta$, we give an explicit formula for the Sprague-Grundy functions of $\beta$-saturations of misère Nim.

\subsection{Mixed-radix number systems}

We introduce some notation for mixed-radix number systems.

Let $\mathbb{N}$ be the set of nonnegative integers. Throughout this paper, $\beta$ denotes a sequence $\left(\beta_{L}\right)_{L \in \mathbb{N}} \in \mathbb{N}^{\mathbb{N}}$ with $\beta_{L} \geqslant 2$ for every $L \in \mathbb{N}$. Define $\beta^{L}=\beta_{0} \cdot \beta_{1} \cdots \beta_{L-1}$. For example, if $\beta=(2,3,2, \ldots)$, then $\beta^{0}=1, \beta^{1}=2$, and $\beta^{2}=6$.

Let $n \in \mathbb{N}$. We denote by $n_{L}^{\beta}$ the $L$ th digit in the mixed base $\beta$ expansion of $n$, that is, if $n_{\geqslant L}^{\beta}$ is the integer quotient ${ }^{3}$ of $n$ divided by $\beta^{L}$, then $n_{L}^{\beta}=n_{\geqslant L}^{\beta} \bmod \beta_{L}$, where $n_{\geqslant L}^{\beta} \bmod \beta_{L}$ is the remainder of $n_{\geqslant L}^{\beta}$ divided by $\beta_{L}$. By definition,

$$
n=\sum_{L \in \mathbb{N}} n_{L}^{\beta} \beta^{L} \quad \text { and } \quad n_{L}^{\beta} \in\left\{0,1, \ldots, \beta_{L}-1\right\}
$$

For example, if $\beta_{L}=b$ for every $L \in \mathbb{N}$, then $n_{L}^{\beta}$ is the $L$ th digit in the ordinary base $b$ expansion of $n$, so it is convenient to write $\beta=b$. For a negative integer $n$, we define $n_{L}^{\beta}$ similarly; then

$$
n_{L}^{\beta}+(-n-1)_{L}^{\beta}=\beta_{L}-1
$$

For example, $(-1)_{L}^{\beta}=\beta_{L}-1$. We drop the superscript $\beta$ when no confusion can arise.

For $n \in \mathbb{Z}$, define

$$
\operatorname{ord}_{\beta}(n)= \begin{cases}\min \left\{L \in \mathbb{N}: n_{L} \neq 0\right\} \quad\left(=\max \left\{L \in \mathbb{N}: \beta^{L} \text { divides } n\right\}\right) & \text { if } n \neq 0 \\ \infty & \text { if } n=0\end{cases}
$$

For example, if $\beta=(3,2,5,4, \ldots)$, then $\operatorname{ord}_{\beta}(54)=\operatorname{ord}_{\beta}\left(4 \cdot \beta^{2}+\beta^{3}\right)=2$.

\footnotetext{
${ }^{1}$ By contrast, an explicit formula is known for the misère Sprague-Grundy function of Nim. See Remark 5.

${ }^{2}$ The concept of saturations was first introduced in [9] to connect Welter's game with representations of symmetric groups.

${ }^{3} n_{\geqslant L}^{\beta}$ is the unique integer satisfying $n-n_{\geqslant L}^{\beta} \beta^{L} \in\left\{0,1, \ldots, \beta^{L}-1\right\}$.
} 


\subsection{Subtraction games}

We define subtraction games, their misère versions, and Sprague-Grundy functions.

Fix a positive integer $k$ and let $\Omega$ denote $\{0,1, \ldots, k-1\}$. Let $\mathcal{P} \subseteq \mathbb{N}^{k}$ and $\mathscr{C} \subseteq$ $\mathbb{N}^{k} \backslash\{(0, \ldots, 0)\}$. Define $\Gamma(\mathcal{P}, \mathscr{C})$ to be the digraph with vertex set $\mathcal{P}$ and edge set

$$
\left\{(X, Y) \in \mathcal{P}^{2}: X-Y \in \mathscr{C}\right\} .
$$

We call $\Gamma(\mathcal{P}, \mathscr{C})$ a subtraction game or a take-away game. The vertex set $\mathcal{P}$ is called the position set of $\Gamma(\mathcal{P}, \mathscr{C})$.

Remark 1 . We can consider $\Gamma(\mathcal{P}, \mathscr{C})$ as a two-player game as follows. Before the game begins, we pick an initial position $X_{0} \in \mathcal{P}$. The first player subtracts some $C_{0} \in \mathscr{C}$ from $X_{0}$ so that $X_{0}-C_{0} \in \mathcal{P}$. Let $X_{1}=X_{0}-C_{0}$. Similarly, the second player subtracts some $C_{1} \in \mathscr{C}$ from $X_{1}$ so that $X_{1}-C_{1} \in \mathcal{P}$. In this way, the two players alternately subtract some $C \in \mathscr{C}$ from the current position. The player who subtracts last wins.

Example 2 (Nim). Let

$$
\mathscr{C}_{[1]}=\left\{C \in \mathbb{N}^{k}: \operatorname{wt}(C)=1\right\},
$$

where $\operatorname{wt}(C)$ is the Hamming weight of $C$, that is, the number of nonzero components of $C$. The subtraction game $\Gamma\left(\mathbb{N}^{k}, \mathscr{C}_{[1]}\right)$ is called $\mathrm{Nim}$. For example, in Nim, the first player will win if we start from $(1,0)$; indeed, he can subtract $(1,0) \in \mathscr{C}_{[1]}$ from $(1,0)$, but the second player cannot subtract any $C \in \mathscr{C}_{[1]}$ from $(0,0)$.

We next define the misère version of a subtraction game. Let $X$ be a position in a subtraction game $\Gamma(\mathcal{P}, \mathscr{C})$. If $X-C \in \mathcal{P}$ for some $C \in \mathscr{C}$, then $X-C$ is called an option of $X$ (in $\Gamma(\mathcal{P}, \mathscr{C})$ ). If $X$ has no options, then $X$ is called a terminal position. Let $\mathcal{P}^{\prime}$ be the set of non-terminal positions in $\Gamma(\mathcal{P}, \mathscr{C})$. The subtraction game $\Gamma\left(\mathcal{P}^{\prime}, \mathscr{C}\right)$ is called the misère version of $\Gamma(\mathcal{P}, \mathscr{C})[11]$.

Example 3 (misère Nim). Let $\mathcal{P}_{\text {Mis }}=\mathcal{P}_{\text {Mis }}^{k}=\mathbb{N}^{k} \backslash\{(0, \ldots, 0)\}$. Then $\mathcal{P}_{\text {Mis }}$ is the set of non-terminal positions of Nim, so the misère version of $\mathrm{Nim}$ is $\Gamma\left(\mathcal{P}_{\text {Mis }}, \mathscr{C}_{[1]}\right)$. We call $\Gamma\left(\mathcal{P}_{\text {Mis }}, \mathscr{C}_{[1]}\right)$ misère Nim. ${ }^{4}$ In misère Nim, the first player will lose if we start from $(1,0)$ because this position is terminal.

We now define Sprague-Grundy functions. See, for example, $[1,2,5,14]$ for details. Let $\Gamma=\Gamma(\mathcal{P}, \mathscr{C})$ and $X \in \mathcal{P}$. The Sprague-Grundy value $\operatorname{sg}(X)$ of $X$ is defined to be the minimum nonnegative integer $n$ such that $n$ is not equal to the Sprague-Grundy value of any option of $X$, that is,

$$
\operatorname{sg}(X)=\operatorname{sg}_{\Gamma}(X)=\operatorname{mex}\left\{\operatorname{sg}_{\Gamma}(Y): Y \text { is an option of } X\right\},
$$

\footnotetext{
${ }^{4}$ Misère Nim is usually defined to be Nim in misère play, so the definition of misère Nim used in this paper is slightly different from the standard one. However, for $X \in \mathbb{N}^{k} \backslash\{(0, \ldots, 0)\}$, the outcomes of $X$ in the two of these misère Nim are the same. In other words, when the initial position is $X$, the first player can win in one of the two misère Nim if and only if he can win in the other misère Nim.
} 
where mex $S=\min \{n \in \mathbb{N}: n \notin S\}$. Note that if $X$ is a terminal position, then $\operatorname{sg}(X)=$ $\operatorname{mex} \emptyset=0$. The nonnegative integer-valued function $\mathrm{sg}: \mathcal{P} \ni X \mapsto \operatorname{sg}(X) \in \mathbb{N}$ is called the Sprague-Grundy function of the subtraction game $\Gamma$. For a position $X$, the following two statements are equivalent:

(1) $\operatorname{sg}(X)=0$.

(2) The second player can win when the initial position is $X$.

Example 4. Let $k=2$. We calculate the Sprague-Grundy values of some positions in misère Nim (see Table 1 in Example 7). Let $\mathcal{G}$ be the Sprague-Grundy function of misère Nim, that is, $\mathcal{G}(X)=\operatorname{sg}_{\Gamma\left(\mathcal{P}_{\mathrm{Mis}}, \mathscr{C}_{[1]}\right)}(X)$ for $X \in \mathcal{P}_{\text {Mis. }}$. Since $(0,1)$ and $(1,0)$ are terminal positions, it follows that $\mathcal{G}((0,1))=\mathcal{G}((1,0))=0$. Hence $\mathcal{G}((0,2))=\mathcal{G}((1,1))=$ $\mathcal{G}((2,0))=\operatorname{mex}\{0\}=1$. This implies that $\mathcal{G}((1,2))=\mathcal{G}((2,1))=\operatorname{mex}\{0,1\}=2$, so $\mathcal{G}((2,2))=\operatorname{mex}\{1,2\}=0$. We can verify that the second player can win when $(2,2)$ is the initial position.

Remark 5. The Sprague-Grundy function $\mathcal{G}$ of misère Nim is different from the misère Sprague-Grundy function $\mathcal{G}^{-}$of Nim defined in [5]. The domain of $\mathcal{G}$ is $\mathbb{N}^{k} \backslash\{(0, \ldots, 0)\}$ and that of $\mathcal{G}^{-}$is $\mathbb{N}^{k}$. Here, for $X \in \mathbb{N}^{k}$, we can compute $\mathcal{G}^{-}(X)$ as follows:

$$
\mathcal{G}^{-}(X)= \begin{cases}1 & \text { if } X=(0, \ldots, 0), \\ \operatorname{mex}\left\{\mathcal{G}^{-}(Y): Y \text { is an option of } X\right\} & \text { otherwise. }\end{cases}
$$

The value $\mathcal{G}^{-}(X)$ is generally not equal to $\mathcal{G}(X)$. For example, $\mathcal{G}^{-}((0,2))=2 \neq 1=$ $\mathcal{G}((0,2))$. However, $\mathcal{G}^{-}(X)=0$ if and only if $\mathcal{G}(X)=0 .^{5}$

\section{$1.3 \quad \beta$-Saturations}

We define $\beta$-saturations of subtraction games.

Elements in $\mathbb{N}^{k}$ will be denoted by upper-case letters, and components of them by lower-case letters with superscripts. For example, $C=\left(c^{0}, \ldots, c^{k-1}\right) \in \mathbb{N}^{k}$. Define

$$
\mathscr{C}^{\beta}=\mathscr{C}^{\beta, k}=\left\{C \in \mathbb{N}^{k} \backslash\{(0, \ldots, 0)\}: \operatorname{ord}_{\beta}\left(\sum_{i \in \Omega} c^{i}\right)=\operatorname{mord}_{\beta}(C)\right\},
$$

where

$$
\operatorname{mord}_{\beta}(C)=\min \left\{\operatorname{ord}_{\beta}\left(c^{i}\right): i \in \Omega\right\} .
$$

For example, $(2,2,6) \in \mathscr{C}^{2}$ and $(2,2,4) \notin \mathscr{C}^{2}$ because

$\operatorname{ord}_{2}(2+2+6)=1=\operatorname{mord}_{2}((2,2,6)) \quad$ and $\quad \operatorname{ord}_{2}(2+2+4)=3>1=\operatorname{mord}_{2}((2,2,4))$.

A subtraction game $\Gamma(\mathcal{P}, \mathscr{C})$ is said to be $\beta$-saturated if its Sprague-Grundy function is equal to that of $\Gamma\left(\mathcal{P}, \mathscr{C}^{\beta}\right)$. If $\Gamma(\mathcal{P}, \mathscr{C})$ is $\beta$-saturated, then we also say that it is a $\beta$-saturation of $\Gamma\left(\mathcal{P}, \mathscr{C}_{[1]}\right)$.

\footnotetext{
${ }^{5}$ As we have mentioned, $\mathcal{G}^{-}(X)$ can be written down explicitly. If $\max X \geqslant 2$, then $\mathcal{G}^{-}(X)=\sigma^{2}(X)$, where $\sigma^{2}(X)$ is the Nim sum of the components of $X$. If $\max X<2$, then $\mathcal{G}^{-}(X)=1-\sigma^{2}(X)$.
} 
Example 6 (Nim and Welter's game). Let

$$
\mathcal{P}_{\mathrm{Wel}}=\left\{X \in \mathbb{N}^{k}: x^{i} \neq x^{j} \text { whenever } i \neq j\right\} .
$$

The subtraction game $\Gamma\left(\mathcal{P}_{\text {Wel }}, \mathscr{C}_{[1]}\right)$ is called Welter's game. It is known that Nim and Welter's game are 2-saturated $[3,6,9,16]$, that is, for $\mathcal{P} \in\left\{\mathbb{N}^{k}, \mathcal{P}_{\text {Wel }}\right\}$, the Sprague-Grundy function of $\Gamma\left(\mathcal{P}, \mathscr{C}_{[1]}\right)$ is equal to that of $\Gamma\left(\mathcal{P}, \mathscr{C}^{2}\right)$. Moreover, $\Gamma\left(\mathbb{N}^{k}, \mathscr{C}\right)$ is 2 -saturated if and only if $\mathscr{C}_{[1]} \subseteq \mathscr{C} \subseteq \mathscr{C}^{2}[3]$.

Example 7. Let $k=2$. We compare the Sprague-Grundy function of misère Nim $\Gamma\left(\mathcal{P}_{\text {Mis }}, \mathscr{C}_{[1]}\right)$ with that of $\Gamma\left(\mathcal{P}_{\text {Mis }}, \mathscr{C}^{2}\right)$ (see Table 1$)$. We first consider the position $(2,2) \in$ $\mathcal{P}_{\text {Mis }}\left(=\mathbb{N}^{2} \backslash\{(0,0)\}\right)$. The Sprague-Grundy value of $(2,2)$ equals 0 in $\Gamma\left(\mathcal{P}_{\text {Mis }}, \mathscr{C}_{[1]}\right) ;$ however, it equals 3 in $\Gamma\left(\mathcal{P}_{\text {Mis }}, \mathscr{C}^{2}\right)$. Indeed, since $(2,2)-(0,1)=(2,1) \in \mathscr{C}^{2}$, it follows that $(0,1)$ is an option of $(2,2)$ in $\Gamma\left(\mathcal{P}_{\text {Mis }}, \mathscr{C}^{2}\right)$. Hence $\operatorname{sg}_{\Gamma\left(\mathcal{P}_{\mathrm{Mis}}, \mathscr{C}^{2}\right)}((2,2))=\operatorname{mex}\{0,1,2\}=3$ (see Table 1). Thus misère Nim is not 2 -saturated when $k=2$. We next compute the Sprague-Grundy value of $(2,3)$ in $\Gamma\left(\mathcal{P}_{\text {Mis }}, \mathscr{C}^{2}\right)$. Since $(2,3)-(0,1)=(2,2) \notin \mathscr{C}^{2}$ and $(2,3)-(1,0)=(1,3) \notin \mathscr{C}^{2},(2,3)$ has no options with Sprague-Grundy value 0, and hence its Sprague-Grundy value is 0 .

Table 1: Sprague-Grundy values in $\Gamma\left(\mathcal{P}_{\text {Mis }}, \mathscr{C}_{[1]}\right)$ and $\Gamma\left(\mathcal{P}_{\text {Mis }}, \mathscr{C}^{2}\right)$.

\begin{tabular}{|c|c|c|c|c|c|c|c|c|c|c|c|c|c|c|c|c|c|c|c|}
\hline & 0 & 1 & 2 & 3 & 4 & 5 & 6 & 7 & 8 & & 0 & 1 & 2 & 3 & 4 & 5 & 6 & 7 & 8 \\
\hline 0 & & 0 & 1 & 2 & 3 & 4 & 5 & 6 & 7 & 0 & & 0 & 1 & 2 & 3 & 4 & 5 & 6 & 7 \\
\hline 1 & 0 & 1 & 2 & 3 & 4 & 5 & 6 & 7 & 8 & 1 & 0 & 1 & 2 & 3 & 4 & 5 & 6 & 7 & 8 \\
\hline 2 & 1 & 2 & 0 & 4 & 5 & 3 & 7 & 8 & 6 & 2 & 1 & 2 & 3 & 0 & 5 & 6 & 7 & 4 & 9 \\
\hline 3 & 2 & 3 & 4 & 0 & 1 & 6 & 8 & 5 & 9 & 3 & 2 & 3 & 0 & 1 & 6 & 7 & 4 & 5 & 10 \\
\hline 4 & 3 & 4 & 5 & 1 & 0 & 2 & 9 & 10 & 11 & 4 & 3 & 4 & 5 & 6 & 7 & 0 & 1 & 2 & 11 \\
\hline 5 & 4 & 5 & 3 & 6 & 2 & 0 & 1 & 9 & 10 & 5 & 4 & 5 & 6 & 7 & 0 & 1 & 2 & 3 & 12 \\
\hline 6 & 5 & 6 & 7 & 8 & 9 & 1 & 0 & 2 & 3 & 6 & 5 & 6 & 7 & 4 & 1 & 2 & 3 & 0 & 13 \\
\hline 7 & 6 & 7 & 8 & 5 & 10 & 9 & 2 & 0 & 1 & 7 & 6 & 7 & 4 & 5 & 2 & 3 & 0 & 1 & 14 \\
\hline 8 & 7 & 8 & 6 & 9 & 11 & 10 & 3 & 1 & 0 & 8 & 7 & 8 & 9 & 10 & 11 & 12 & 13 & 14 & 15 \\
\hline
\end{tabular}

\subsection{A formula for $\beta$-saturations of misère Nim}

We present an explicit formula for the Sprague-Grundy functions of $\beta$-saturations of misère Nim.

Let $\llbracket \beta_{L} \rrbracket$ denote $\left\{0,1, \ldots, \beta_{L}-1\right\}$ equipped with the following two operations: for $a, b \in \llbracket \beta_{L} \rrbracket$,

$$
a \oplus b=(a+b) \bmod \beta_{L} \quad \text { and } \quad a \ominus b=(a-b) \bmod \beta_{L} .
$$

In other words, $\llbracket \beta_{L} \rrbracket$ is the additive group of integers modulo $\beta_{L}$. For example, in $\llbracket 5 \rrbracket$, $2 \oplus 4=1$ and $2 \ominus 4=3$. For $n \in \mathbb{N}$, we will think of $n_{L}^{\beta}$ as an element of $\llbracket \beta_{L} \rrbracket$. Let $\mathbb{Z}_{\beta}$ denote $\prod_{L \in \mathbb{N}} \llbracket \beta_{L} \rrbracket$ equipped with the following two operations: for $n, m \in \mathbb{Z}_{\beta}$,

$$
n \oplus m=\left[n_{L} \oplus m_{L}\right]_{L \in \mathbb{N}} \quad \text { and } \quad n \ominus m=\left[n_{L} \ominus m_{L}\right]_{L \in \mathbb{N}},
$$


where $n=\left[n_{L}\right]_{L \in \mathbb{N}}$ and $m=\left[m_{L}\right]_{L \in \mathbb{N}}$. For $n \in \mathbb{Z}_{\beta}$, define

$$
\operatorname{ord}_{\beta}(n)= \begin{cases}\min \left\{L \in \mathbb{N}: n_{L} \neq 0\right\} & \text { if } n \neq[0,0, \ldots] \\ \infty & \text { if } n=[0,0, \ldots]\end{cases}
$$

Consider the map $\Phi: \mathbb{Z} \ni n \mapsto\left[n_{L}^{\beta}\right]_{L \in \mathbb{N}} \in \mathbb{Z}_{\beta}$. For $n \in \mathbb{Z}$, we identify $\Phi(n)$ with $n$. Let $\mathcal{N}_{\beta}$ denote $\Phi(\mathbb{N})$. For $n \in \mathcal{N}_{\beta}$, it is convenient to write $n=\left[n_{0}, n_{1}, \ldots, n_{L-1}\right]$ when $n_{\geqslant L}=0$. For example, if $\beta=10$, then $24=[4,2,0, \ldots]=[4,2] \in \mathcal{N}_{10}$.

For $X \in \mathcal{N}_{\beta}^{k}$, define

$$
\sigma^{\beta}(X)=\sigma^{\beta, k}(X)=x^{0} \oplus \cdots \oplus x^{k-1}\left(\in \mathcal{N}_{\beta}\right) .
$$

Let $\sigma_{L}^{\beta}(X)=\left(\sigma^{\beta}(X)\right)_{L}\left(=\right.$ the $L$ th digit of $\left.\sigma^{\beta}(X)\right)$ for $L \in \mathbb{N}$.

Example 8 ( $\beta$-saturations of Nim [10]). Let $X$ be a position in a $\beta$-saturation of Nim. Then

$$
\operatorname{sg}(X)=\sigma^{\beta}(X) \text {. }
$$

For example, if $\beta=(3,2,5, \ldots)$ and $X=(16,27)=([1,1,2],[0,1,4]) \in \mathcal{N}_{\beta}^{2}$, then

$$
\operatorname{sg}(X)=\sigma^{\beta}(X)=[1 \oplus 0,1 \oplus 1,2 \oplus 4]=[1,0,1]=7 .
$$

Example 9 (b-saturations of Welter's game [9]). Let $X$ be a position in a $b$-saturation of Welter's game, where $b$ is an integer greater than 1 . Then

$$
\operatorname{sg}(X)=\sigma^{b}(X) \oplus \bigoplus_{i<j}\left(b^{\operatorname{ord}_{b}\left(x^{i}-x^{j}\right)+1}-1\right) .
$$

For example, if $b=3$ and $X=(1,4) \in \mathcal{N}_{3}^{2}$, then $\operatorname{sg}(X)=1 \oplus 4 \oplus\left(3^{2}-1\right)=1$.

We now give an explicit formula for the Sprague-Grundy functions of $\beta$-saturations of misère Nim. For $X \in \mathcal{P}_{\text {Mis }}\left(\subseteq \mathcal{N}_{\beta}^{k}\right)$, define

$$
\phi^{\beta}(X)=\sigma^{\beta}(X) \oplus\left(\beta^{\operatorname{mord}_{\beta}(X)+1}-1\right) .
$$

Theorem 10. The Sprague-Grundy function of a $\beta$-saturation of misère Nim is equal to $\phi^{\beta}$, that is,

$$
\operatorname{sg}(X)=\phi^{\beta}(X)
$$

for every position $X$ in a $\beta$-saturation of misère Nim.

Before giving an example of Theorem 10, we introduce some notation. For $n \in \mathcal{N}_{\beta}$ and $L \in \mathbb{N}$, let $n_{<L}=\left[n_{0}, n_{1}, \ldots, n_{L-1}\right] \in \mathcal{N}_{\beta}$, that is, $n_{<L}=n \bmod \beta^{L}$. For $X \in \mathcal{N}_{\beta}^{k}$, let $X_{<L}=\left(x_{<L}^{0}, x_{<L}^{1} \ldots, x_{<L}^{k-1}\right) \in \mathcal{N}_{\beta}^{k}$. When $X=X_{<L}$, it is convenient to write $X$ as follows:

$$
X=\left[\begin{array}{cccc}
x_{0}^{0} & x_{1}^{0} & \cdots & x_{L-1}^{0} \\
\vdots & \vdots & \ddots & \vdots \\
x_{0}^{k-1} & x_{1}^{k-1} & \cdots & x_{L-1}^{k-1}
\end{array}\right]
$$


Note that if $\operatorname{mord}_{\beta}(X)=N$, then

$$
X=\left[\begin{array}{cccc} 
& x_{N}^{0} & \cdots & x_{L-1}^{0} \\
0 & \vdots & \cdots & \vdots \\
& x_{N}^{k-1} & \cdots & x_{L-1}^{k-1}
\end{array}\right]
$$

Example 11. Let us consider Example 7 again. Let

$$
X=(2,2)=\left[\begin{array}{ll}
0 & 1 \\
0 & 1
\end{array}\right] \in \mathcal{N}_{2}^{2} \quad \text { and } \quad Y=(2,3)=\left[\begin{array}{ll}
0 & 1 \\
1 & 1
\end{array}\right] \in \mathcal{N}_{2}^{2} .
$$

Then $\operatorname{mord}_{2}(X)=1$ and $\operatorname{mord}_{2}(Y)=0$, so

$$
\phi^{2}(X)=\sigma^{2}(X) \oplus\left(2^{1+1}-1\right)=3 \quad \text { and } \quad \phi^{2}(Y)=\sigma^{2}(Y) \oplus\left(2^{0+1}-1\right)=0 .
$$

\subsection{The weight of $\phi^{\beta, k}$}

We give the minimum of the weight of $\mathscr{C}$ such that $\Gamma\left(\mathcal{P}_{\text {Mis }}, \mathscr{C}\right)$ is a $\beta$-saturation of misère Nim.

Let $\mathcal{P} \subseteq \mathbb{N}^{k}$. For a nonnegative integer-valued function $\psi: \mathcal{P} \rightarrow \mathbb{N}$, let $\Delta(\psi)$ be the set of $\mathscr{C} \subseteq \mathbb{N}^{k} \backslash\{(0, \ldots, 0)\}$ such that the Sprague-Grundy function of $\Gamma(\mathcal{P}, \mathscr{C})$ equals $\psi$. Note that if $\mathscr{C}, \mathscr{D} \in \Delta(\psi)$ and $\mathscr{C} \subseteq \mathscr{E} \subseteq \mathscr{D}$, then $\mathscr{E} \in \Delta(\psi)$. By definition, $\Gamma(\mathcal{P}, \mathscr{C})$ is $\beta$-saturated if and only if $\mathscr{C} \in \Delta\left(\psi^{\beta}\right)$, where $\psi^{\beta}$ is the Sprague-Grundy function of $\Gamma\left(\mathcal{P}, \mathscr{C}^{\beta}\right)$. If $\Delta(\psi) \neq \emptyset$, then define

$$
\mathrm{wt}(\psi)=\min _{\mathscr{C} \in \Delta(\psi)} \mathrm{wt}(\mathscr{C}),
$$

where $\operatorname{wt}(\mathscr{C})=\max \{\operatorname{wt}(C): C \in \mathscr{C}\}$ and $\max \emptyset=0$. For example, if $\psi^{2}$ is the SpragueGrundy function of a 2-saturation of Nim or that of a 2-saturation of Welter's game, then

$$
\mathrm{wt}\left(\psi^{2}\right)=1
$$

since $\mathscr{C}_{[1]} \in \Delta\left(\psi^{2}\right)$. In other words, Nim and Welter's game themselves are 2-saturated. However, as we have seen in Example 7, if $k=2$, then $\mathscr{C}_{[1]} \notin \Delta\left(\phi^{2,2}\right)$, so wt $\left(\phi^{2,2}\right)=2$. In fact, if $k \geqslant 2$, then

$$
\mathrm{wt}\left(\phi^{2, k}\right)=2 .
$$

Let $B$ be the supremum of $\left\{\beta_{L}: L \geqslant 1\right\}$ in $\mathbb{N} \cup\{\infty\}$. In general, we will prove that

$$
\begin{aligned}
\operatorname{wt}\left(\phi^{\beta, k}\right) & =\max \left\{\min \left\{\beta_{L}-\delta(L), k-\delta(L)\left[\beta_{0}<2 k\right]\right\}: L \in \mathbb{N}\right\} \\
& = \begin{cases}k & \text { if } B \geqslant k \text { or } \beta_{0} \geqslant 2 k, \\
k-1 & \text { if } B<k \text { and } k \leqslant \beta_{0}<2 k, \\
\max \left\{\beta_{0}-1, B\right\} & \text { if } B<k \text { and } \beta_{0}<k,\end{cases}
\end{aligned}
$$

where $\delta(L)=[L=0]$ and [ ] is the Iverson bracket notation, that is, $[P]=1$ if a statement $P$ holds, and $[P]=0$ otherwise. In particular, if $\beta=b$ for some $b \in \mathbb{N}$, then

$$
\operatorname{wt}\left(\phi^{b, k}\right)=\min \{b, k\} \text {. }
$$




\section{Proofs}

When no confusion can arise, we write $\sigma$ and $\phi$ instead of $\sigma^{\beta}$ and $\phi^{\beta}$, respectively.

\subsection{Preliminaries}

Let $\mathscr{C} \subseteq \mathcal{N}_{\beta}^{k} \backslash\{(0, \ldots, 0)\}$. The Sprague-Grundy function of $\Gamma\left(\mathcal{P}_{\text {Mis }}, \mathscr{C}\right)$ equals $\phi$ if and only if $\mathscr{C}$ satisfies the following two conditions:

(SG1) If $X \in \mathcal{P}_{\text {Mis }}$, then $X$ has no option $Y$ with $\phi(Y)=\phi(X)$ in $\Gamma\left(\mathcal{P}_{\text {Mis }}, \mathscr{C}\right)$, that is, $\phi(X-C) \neq \phi(X)$ for every $C \in \mathscr{C}$ with $X-C \in \mathcal{P}_{\text {Mis }}$.

(SG2) If $X \in \mathcal{P}_{\text {Mis }}$ and $0 \leqslant m<\phi(X)$, then $X$ has an option $Y$ with $\phi(Y)=m$ in $\Gamma\left(\mathcal{P}_{\text {Mis }}, \mathscr{C}\right)$, that is, $\phi(X-C)=m$ for some $C \in \mathscr{C}$ with $X-C \in \mathcal{P}_{\text {Mis }}$.

To prove Theorem 10 and (1.3), it therefore suffices to show the following three assertions:

(A1) $\mathscr{C}^{\beta}$ satisfies (SG1).

(A2) $\left\{C \in \mathcal{N}_{\beta}^{k}: \operatorname{wt}(C)<w\right\}$ does not satisfy (SG2), where $w$ is the right-hand side of (1.3).

(A3) $\mathscr{C}_{[w]}^{\beta}$ satisfies (SG2), where $\mathscr{C}_{[w]}^{\beta}=\left\{C \in \mathscr{C}^{\beta}: \mathrm{wt}(C) \leqslant w\right\}$.

\subsection{Proof of (A1)}

Let $X \in \mathcal{P}_{\text {Mis }}, C \in \mathscr{C}^{\beta}$ with $X-C \in \mathcal{P}_{\text {Mis }}$, and $Y=X-C$. Let $N=\operatorname{mord}_{\beta}(X), M=$ $\operatorname{mord}_{\beta}(Y)$, and $H=\operatorname{mord}_{\beta}(C)$. We show that $\phi_{H}(Y) \neq \phi_{H}(X)$, where $\phi_{H}(X)=$ $(\phi(X))_{H}$. Since $\left(\beta^{N+1}-1\right)_{L}=\ominus[L \leqslant N]$ for $L \in \mathbb{N}$, it follows that

$$
\phi_{L}(X)=\sigma_{L}(X) \ominus[L \leqslant N] \quad \text { and } \quad \phi_{L}(Y)=\sigma_{L}(Y) \ominus[L \leqslant M] .
$$

We first show that

$$
[H \leqslant N]=[H \leqslant M]
$$

Since $C_{<H}=(0, \ldots, 0)$, we see that $Y_{<H}=(X-C)_{<H}=X_{<H}$. Suppose that $H>N$. Then $Y_{\leqslant N}=X_{\leqslant N} \neq(0, \ldots, 0)$, where $Y_{\leqslant N}=Y_{<N+1}$. Hence $M=N$, so (2.2) holds. If $H \leqslant N$, then $Y_{<H}=X_{<H}=(0, \ldots, 0)$, so $H \leqslant M$. Therefore (2.2) holds.

We next show that

$$
\sigma_{H}(X) \neq \sigma_{H}(Y)
$$

Since $C_{<H}=(0, \ldots, 0)$, it follows that $y_{H}^{i}=x_{H}^{i} \ominus c_{H}^{i}$ for $i \in \Omega$. Hence

$$
\sigma_{H}(Y)=\sigma_{H}(X) \ominus \sigma_{H}(C) .
$$

Recall that $H=\operatorname{mord}_{\beta}(C)=\operatorname{ord}_{\beta}\left(\sum_{i} c^{i}\right)$ since $C \in \mathscr{C}^{\beta}$. This implies that $\left(\sum_{i} c^{i}\right)_{H}=$ $\sigma_{H}(C) \neq 0$. Thus $(2.3)$ holds.

Combining (2.1)-(2.3), we see that $\phi_{H}(X) \neq \phi_{H}(Y)$. Therefore $\mathscr{C}^{\beta}$ satisfies (SG1). 


\subsection{Proof of (A2)}

A position $Y \in \mathcal{P}_{\text {Mis }}$ is called a descendant of a position $X \in \mathcal{P}_{\text {Mis }}$ if $X-Y \in \mathcal{N}_{\beta}^{k}$.

If $k=1$, then $w=1$, so (A2) is obvious. Suppose that $k \geqslant 2$. It suffices to show that there exist $X \in \mathcal{P}_{\text {Mis }}$ and $m$ with $0 \leqslant m<\phi(X)$ satisfying the following condition: if $Y$ is a descendant of $X$ with $\phi(Y)=m$, then $\operatorname{wt}(X-Y) \geqslant w$. By (1.3),

$$
w=\min \left\{\beta_{N}-\delta(N), k-\delta(N)\left[\beta_{0}<2 k\right]\right\} \text { for some } N \in \mathbb{N} .
$$

Note that $w \geqslant 1$. We divide the proof into two cases.

Case $1\left(\boldsymbol{N}>\mathbf{0}\right.$ or $\left.\boldsymbol{\beta}_{\mathbf{0}}<\mathbf{2} \boldsymbol{k}\right)$. We see that $w=\min \left\{\beta_{N}, k\right\}-\delta(N)$. Let

$$
X=(\underbrace{\beta^{N}, \ldots, \beta^{N}}_{w+\delta(N)}, 0, \ldots, 0) \in \mathcal{P}_{\text {Mis }}^{k} .
$$

Since $w+\delta(N) \leqslant \beta_{N}$ and $\operatorname{mord}_{\beta}(X)=N$, it follows that

$$
\begin{aligned}
\phi(X) & =\sigma(X) \oplus\left(\beta^{N+1}-1\right)=[\overbrace{0, \ldots, 0}^{N}, \underbrace{1 \oplus \cdots \oplus 1}_{w+\delta(N)}] \ominus[\overbrace{1, \ldots, 1}^{1}, 1] \\
& = \begin{cases}w \beta^{N}-1 & \text { if } \quad N>0, \\
w & \text { if } \quad N=0 .\end{cases}
\end{aligned}
$$

In particular, $\phi(X)>0$. Let $Y$ be a descendant of $X$ with $\phi(Y)=0$. To prove that $\operatorname{wt}(X-Y)=w$, we show that $\sum_{i} y_{N}^{i}=\delta(N)$. Since $\phi_{0}(Y)=0$, we see that $\operatorname{mord}_{\beta}(Y)=0$. Hence $\phi_{N}(Y)=\sigma_{N}(Y) \ominus[N \leqslant 0]=\sigma_{N}(Y) \ominus \delta(N)$. Since $\phi_{N}(Y)=0$,

$$
\sigma_{N}(Y)=\delta(N)
$$

We also see that $\sum_{i} y_{N}^{i}<\sum_{i} x_{N}^{i}=w+\delta(N) \leqslant \beta_{N}$ because $Y$ is a descendant of $X$ with $Y \neq X$. Hence $\sum_{i} y_{N}^{i}=\sigma_{N}(Y)=\delta(N)$. Therefore $\operatorname{wt}(X-Y)=w$.

Case $2\left(\boldsymbol{N}=\mathbf{0}\right.$ and $\left.\boldsymbol{\beta}_{\mathbf{0}} \geqslant \mathbf{2} \boldsymbol{k}\right)$. Since $k \geqslant 2$, we see that $\beta_{0} \geqslant 4$ and $w=\min \left\{\beta_{0}-1, k\right\}=$ $k$. Let

$$
X=(2, \ldots, 2) \in \mathcal{P}_{\text {Mis }}^{k} .
$$

Then $\phi(X)=\phi_{0}(X)=2 k-1>0$. Let $Y$ be a descendant of $X$ with $\phi(Y)=0$. Then $\phi_{0}(Y)=\sigma_{0}(Y) \ominus 1=0$. Since $\sum_{i} y_{0}^{i}<\sum_{i} x_{0}^{i}=2 k \leqslant \beta_{0}$, it follows that $\sum_{i} y_{0}^{i}=\sigma_{0}(Y)=1$. This implies that $y^{i} \in\{0,1\}$ for $i \in \Omega$. Hence $\operatorname{wt}(X-Y)=k=w$.

Example 12. Let $\beta=(6,2,2, \ldots), k=3$, and $X=(2,2,2)$. Then $\phi(X)=5$. If $Y$ is a descendant of $X$ with $\phi(Y)=0$, then $Y \in\{(0,0,1),(0,1,0),(1,0,0)\}$, and hence $\operatorname{wt}(X-Y)=3$. Note that if $\beta=(5,2,2, \ldots)$, then $\phi((2,2,2))=0$. 


\subsection{Proof of (A3)}

To prove (A3), we present two lemmas.

For $X \in \mathcal{P}_{\text {Mis }}$, the next lemma allows us to express $x^{0}$ with $x^{1}, \ldots, x^{k-1}$, and $\phi(X)$.

Lemma 13. Let $X \in \mathcal{P}_{\text {Mis }}$ and $m \in \mathcal{N}_{\beta}$. For $i \in \Omega$, let

$$
\begin{gathered}
M^{(i)}=\operatorname{mord}_{\beta}\left(\left(m \ominus(-1), x^{0}, \ldots, x^{i-1}, x^{i+1}, \ldots, x^{k-1}\right)\right), \\
y^{(i)}=m \ominus\left(\beta^{M^{(i)}+1}-1\right) \ominus x^{0} \ominus \cdots \ominus x^{i-1} \ominus x^{i+1} \ominus \cdots \ominus x^{k-1},
\end{gathered}
$$

and

$$
Y^{(i)}=\left(x^{0}, \ldots, x^{i-1}, y^{(i)}, x^{i+1}, \ldots, x^{k-1}\right) .
$$

Then $\operatorname{mord}_{\beta}\left(Y^{(i)}\right)=M^{(i)}$. In particular, $\phi\left(Y^{(i)}\right)=m$.

Proof. It suffices to prove the lemma when $i=0$. Let $M=M^{(0)}$ and $Y=Y^{(0)}$. We show that $\operatorname{mord}_{\beta}(Y)=M$. For $L<M$,

$$
\begin{aligned}
y_{L}^{0} & =m_{L} \ominus\left(\beta^{M+1}-1\right)_{L} \ominus x_{L}^{1} \ominus \cdots \ominus x_{L}^{k-1} \\
& =m_{L} \ominus(\ominus 1)=(m \ominus(-1))_{L}=0 .
\end{aligned}
$$

This implies that $\operatorname{mord}_{\beta}(Y) \geqslant M$. By the definition of $M$, we see that $(m \ominus(-1))_{M} \neq 0$ or $x_{M}^{j} \neq 0$ for some $j \geqslant 1$. If the latter holds, then $\operatorname{mord}_{\beta}(Y)=M$. Suppose that $(m \ominus(-1))_{M} \neq 0$ and $x_{M}^{j}=0$ for every $j \geqslant 1$. Then $y_{M}^{0}=m_{M} \ominus\left(\beta^{M+1}-1\right)_{M} \ominus x_{M}^{1} \ominus$ $\cdots \ominus x_{M}^{k-1}=(m \ominus(-1))_{M} \neq 0$, so $\operatorname{mord}_{\beta}(Y)=M$. Therefore

$$
m=y^{0} \oplus x^{1} \oplus \cdots \oplus x^{k-1} \oplus\left(\beta^{M+1}-1\right)=\sigma^{\beta}(Y) \oplus\left(\beta^{\operatorname{mord}_{\beta}(Y)+1}-1\right)=\phi(Y) .
$$

Example 14. Let $\beta=3, X=(3,4)$, and $m=2$. Note that $m \ominus(-1)=[0,1,1, \ldots]$ and $\operatorname{ord}_{3}(m \ominus(-1))=1$. Since

$$
M^{(0)}=\operatorname{mord}_{3}((m \ominus(-1), 4))=0 \text { and } M^{(1)}=\operatorname{mord}_{3}((m \ominus(-1), 3))=1,
$$

it follows that

$$
y^{(0)}=m \ominus\left(3^{1}-1\right) \ominus 4=8 \text { and } y^{(1)}=m \ominus\left(3^{2}-1\right) \ominus 3=0 .
$$

By Lemma 13, $\phi\left(\left(y^{(0)}, x^{1}\right)\right)=\phi\left(\left(x^{0}, y^{(1)}\right)\right)=m=2$. Indeed,

$$
\phi((8,4))=8 \oplus 4 \oplus\left(3^{1}-1\right)=2 \text { and } \phi((3,0))=3 \oplus 0 \oplus\left(3^{2}-1\right)=2 .
$$

The following trivial lemma will be used to construct appropriate options. For $X \in \mathcal{N}_{\beta}^{k}$ and $L \in \mathbb{N}$, let $x_{L}=\left(x_{L}^{0}, x_{L}^{1}, \ldots, x_{L}^{k-1}\right) \in \llbracket \beta_{L} \rrbracket^{k}$. For example, if $\beta=3$ and $X=$ $([1,0,2],[2,1])$, then $x_{0}=(1,2)$ and $x_{1}=(0,1)$.

Lemma 15. Let $X \in \mathcal{N}_{\beta}^{k}, m \in \mathcal{N}_{\beta}$, and $R \in \mathbb{N}$. Choose $j \in \Omega$ so that $x_{R}^{j} \geqslant x_{R}^{i}$ for every $i \in \Omega$. If $m_{R} \leqslant \sum_{i} x_{R}^{i}$, then there exists $u \in \llbracket \beta_{R} \rrbracket^{k}$ satisfying the following three conditions: 
(1) $\bigoplus_{i \in \Omega} u^{i}=m_{R}$, where $u=\left(u^{0}, \ldots, u^{k-1}\right)$.

(2) $0 \leqslant x_{R}^{i}-u^{i} \leqslant x_{R}^{j}-u^{j}$ for every $i \in \Omega$.

(3) $\sum_{i \in \Omega}\left(x_{R}^{i}-u^{i}\right) \leqslant \beta_{R}-1$.

Before proving Lemma 15, let us give an example. Let $\beta=(7,2, \ldots), X=(4,4,3)$, $R=j=0$, and $m=6$. Then $m_{0}=6<11=\sum_{i} x_{0}^{i}$. Since $\left(\oplus_{i} x_{0}^{i}\right) \ominus m_{0}=4 \ominus 6=5<11$, we can obtain $u$ satisfying (1)-(3) by subtracting a vector $\left(t^{0}, t^{1}, t^{2}\right)$ with $\sum t^{i}=5$ from $x_{0}$. For example, let $t=(4,1,0)$; then $x_{0}-t=(0,3,3)$, and it satisfies (1)-(3).

Proof. By rearranging $x^{0}, \ldots, x^{k-1}$ if necessary, we may assume that $j=0$. Let $d=$ $\left(\oplus_{i} x_{R}^{i}\right) \ominus m_{R}$. Since $m_{R} \leqslant \sum_{i} x_{R}^{i}$, it follows that $d \leqslant \sum_{i} x_{R}^{i}-m_{R} \leqslant \sum_{i} x_{R}^{i}$. For $i \in \Omega$, let

$$
t^{i}=\min \left\{x_{R}^{i}, d-\sum_{h=0}^{i-1} t^{h}\right\} .
$$

Then $\sum_{i} t^{i}=d$. It follows that $x_{R}-\left(t^{0}, \ldots, t^{k-1}\right)$ satisfies $(1)-(3)$.

We now prove (A3). Let $n=\phi(X)$ and $m \in \mathcal{N}_{\beta}$ with $0 \leqslant m<n$. We show that $\phi(X-C)=m$ for some $C \in \mathscr{C}_{[w]}^{\beta}$ with $X-C \in \mathcal{P}_{\text {Mis. }}$ Let $N=\operatorname{mord}_{\beta}(X)$ and

$$
R=\max \left\{L \in \mathbb{N}: m_{L} \neq n_{L}\right\} .
$$

We divide the proof into two cases.

Case $1(\boldsymbol{N}>\boldsymbol{R})$. By rearranging $x^{0}, \ldots, x^{k-1}$ if necessary, we may assume that $x_{N}^{0} \neq 0$. For $i \geqslant 1$, let $y^{i}=x^{i}$. Let

$$
\begin{gathered}
M=\operatorname{mord}_{\beta}\left(\left(m \ominus(-1), y^{1}, \ldots, y^{k-1}\right)\right), \\
y^{0}=m \ominus\left(\beta^{M+1}-1\right) \ominus y^{1} \ominus \cdots \ominus y^{k-1},
\end{gathered}
$$

and $Y=\left(y^{0}, y^{1}, \ldots, y^{k-1}\right)$. By Lemma $13, \phi(Y)=m$ and $\operatorname{mord}_{\beta}(Y)=M$. It remains to prove that $y^{0}<x^{0}$. We show that $y_{N}^{0}=x_{N}^{0}-1$ and $y_{\geqslant N+1}^{0}=x_{\geqslant N+1}^{0}$. Since $m_{R}<$ $n_{R} \leqslant \beta_{R}-1$, we see that $(m \ominus(-1))_{R}=m_{R} \oplus 1 \neq 0$. Hence $M \leqslant R<N$. Since $\operatorname{mord}_{\beta}(Y)=M$,

$$
m_{N}=y_{N}^{0} \oplus y_{N}^{1} \oplus \cdots \oplus y_{N}^{k-1} \ominus[N \leqslant M]=y_{N}^{0} \oplus x_{N}^{1} \oplus \cdots \oplus x_{N}^{k-1} .
$$

Moreover, since $N>R$,

$$
m_{N}=n_{N}=x_{N}^{0} \oplus x_{N}^{1} \oplus \cdots \oplus x_{N}^{k-1} \ominus[N \leqslant N]=x_{N}^{0} \oplus x_{N}^{1} \oplus \cdots \oplus x_{N}^{k-1} \ominus 1 .
$$

By (2.5) and (2.6), $y_{N}^{0}=x_{N}^{0} \ominus 1$. Since $x_{N}^{0} \neq 0$, it follows that $y_{N}^{0}=x_{N}^{0} \ominus 1=x_{N}^{0}-1$. Similarly, for $L \geqslant N+1$,

$$
y_{L}^{0} \oplus x_{L}^{1} \oplus \cdots \oplus x_{L}^{k-1} \ominus[L \leqslant M]=m_{L}=n_{L}=x_{L}^{0} \oplus x_{L}^{1} \oplus \cdots \oplus x_{L}^{k-1} \ominus[L \leqslant N] .
$$

Since $[L \leqslant M]=[L \leqslant N]=0$, we see that $y_{L}^{0}=x_{L}^{0}$. Therefore $y^{0}<x^{0}$ and $X-Y=$ $\left(x^{0}-y^{0}, 0, \ldots, 0\right) \in \mathscr{C}_{[w]}^{\beta}$. 
Case $2(\boldsymbol{N} \leqslant \boldsymbol{R})$. By rearranging $x^{0}, \ldots, x^{k-1}$ if necessary, we may assume that $x_{R}^{0} \geqslant$ $x_{R}^{1} \geqslant \cdots \geqslant x_{R}^{k-1}$. Let $M=\operatorname{mord}_{\beta}\left(\left(m \ominus(-1), x^{1}, \ldots, x^{k-1}\right)\right)$. Since $m_{R}<n_{R} \leqslant \beta_{R}-1$, it follows that $M \leqslant R$.

Claim. There exists $u \in \llbracket \beta_{R} \rrbracket^{k}$ satisfying the following four conditions:

(C1) $\bigoplus_{i \in \Omega} u^{i}=m_{R} \oplus[R \leqslant M]$, where $u=\left(u^{0}, \ldots, u^{k-1}\right)$.

(C2) $0 \leqslant x_{R}^{i}-u^{i} \leqslant x_{R}^{0}-u^{0}$ for every $i \in \Omega$.

(C3) $u^{0}<x_{R}^{0}$ unless $N<R=M$.

(C4) $\operatorname{wt}\left(x_{R}-u\right) \leqslant w$.

Assuming the claim for the moment, we construct $Y$ with $\phi(Y)=m$ and $X-Y \in \mathscr{C}_{[w]}^{\beta}$. For $i \geqslant 1$, let

$$
y^{i}=\left[x_{0}^{i}, \ldots, x_{R-1}^{i}, u^{i}, x_{R+1}^{i}, x_{R+2}^{i}, \ldots\right]=x^{i}-\left(x_{R}^{i}-u^{i}\right) \beta^{R}\left(\leqslant x^{i}\right) .
$$

Then

$$
\operatorname{mord}_{\beta}\left(\left(m \ominus(-1), y^{1}, \ldots, y^{k-1}\right)\right)=M .
$$

Indeed, if $M<R$, then $(2.8)$ is obvious. If $M=R$, then $(m \ominus(-1))_{M}=(m \ominus(-1))_{R} \neq 0$, so $(2.8)$ holds. Let

$$
y^{0}=m \ominus\left(\beta^{M+1}-1\right) \ominus y^{1} \ominus \cdots \ominus y^{k-1}
$$

and $Y=\left(y^{0}, y^{1}, \ldots, y^{k-1}\right)$. It follows from (2.8) and Lemma 13 that $\phi(Y)=m$ and $\operatorname{mord}_{\beta}(Y)=M$.

Let $C=X-Y$. We next show that $C \in \mathscr{C}_{[w]}^{\beta}$, that is,

(a) $C \in \mathcal{N}_{\beta}^{k} \backslash\{(0, \ldots, 0)\}$,

(b) $\operatorname{wt}(C) \leqslant w$, and

(c) $\operatorname{ord}_{\beta}\left(\sum c^{i}\right)=\operatorname{mord}_{\beta}(C)$.

(a) Since $\phi(Y)=m \neq n=\phi(X)$, it follows that $C \neq(0, \ldots, 0)$. By $(2.7)$, we see that $y^{i} \leqslant x^{i}$ for $i \geqslant 1$. To prove that $y^{0} \leqslant x^{0}$, we show that $y_{\geqslant R+1}^{0}=x_{\geqslant R+1}^{0}$ and $y_{R}^{0}=u^{0}$. By (2.9), for $L>R(\geqslant M, N)$,

$$
y_{L}^{0}=m_{L} \oplus[L \leqslant M] \ominus y_{L}^{1} \ominus \cdots \ominus y_{L}^{k-1}=n_{L} \ominus x_{L}^{1} \ominus \cdots \ominus x_{L}^{k-1}=x_{L}^{0} \ominus[L \leqslant N]=x_{L}^{0} .
$$

We also see that

$$
y_{R}^{0}=m_{R} \oplus[R \leqslant M] \ominus y_{R}^{1} \ominus \cdots \ominus y_{R}^{k-1}=m_{R} \oplus[R \leqslant M] \ominus u^{1} \ominus \cdots \ominus u^{k-1}=u^{0}
$$

by (C1). Hence if $u^{0}<x_{R}^{0}$, then $y^{0}<x^{0}$. Suppose that $u^{0}=x_{R}^{0}$. Then $M=R$ by (C3). Since $M=\operatorname{mord}_{\beta}(Y)$,

$$
y^{0}=[\underbrace{0, \ldots, 0}_{M}, u^{0}, x_{M+1}^{0}, x_{M+2}^{0}, \ldots]=[\underbrace{0, \ldots, 0}_{M}, x_{M}^{0}, x_{M+1}^{0}, x_{M+2}^{0}, \ldots] \leqslant x^{0} .
$$


(b) If $u^{0} \neq x_{R}^{0}$, then $\operatorname{wt}(C)=\operatorname{wt}\left(x_{R}-u\right)$, and hence $\operatorname{wt}(C) \leqslant w$ by (C4). Suppose that $u^{0}=x_{R}^{0}$. By $(\mathrm{C} 2), u=x_{R}$, so $\operatorname{wt}(C)=1 \leqslant w$.

(c) For $i \geqslant 1$, we know that $c^{i}=x^{i}-y^{i}=\left(x_{R}^{i}-u^{i}\right) \beta^{R}$. Hence if $\operatorname{ord}_{\beta}\left(c^{0}\right)<R$, then

$$
\operatorname{mord}_{\beta}(C)=\operatorname{ord}_{\beta}\left(c^{0}\right)=\operatorname{ord}_{\beta}\left(\sum_{i \in \Omega} c^{i}\right) .
$$

Suppose that $\operatorname{ord}_{\beta}\left(c^{0}\right) \geqslant R$. Then $R \leqslant \operatorname{mord}_{\beta}(C) \leqslant \operatorname{ord}_{\beta}\left(\sum c^{i}\right)$, so we need only show that $\operatorname{ord}_{\beta}\left(\sum c^{i}\right)=R$, that is,

$$
\bigoplus_{i \in \Omega} c_{R}^{i} \neq 0
$$

We first show that $M=N$. Since $Y_{<R}=X_{<R}$ and $M, N \leqslant R$, it follows that if $M<R$ or $N<R$, then $M=N$. If $M \geqslant R$ and $N \geqslant R$, then $M=R=N$. We now show (2.10). Since $y_{R}^{i}=x_{R}^{i} \ominus c_{R}^{i}$, we see that

$$
\begin{aligned}
m_{R}=\bigoplus_{i \in \Omega} y_{R}^{i} \ominus[R \leqslant M] & =\bigoplus_{i \in \Omega} x_{R}^{i} \ominus\left(\bigoplus_{i \in \Omega} c_{R}^{i}\right) \ominus[R \leqslant N] \\
& =n_{R} \ominus\left(\bigoplus_{i \in \Omega} c_{R}^{i}\right) .
\end{aligned}
$$

Since $m_{R} \neq n_{R}$, it follows that (2.10) holds, so $\operatorname{ord}_{\beta}\left(\sum c^{i}\right)=R=\operatorname{mord}_{\beta}(C)$. Therefore $C \in \mathscr{C}_{[w]}^{\beta}$.

It remains to prove the claim. We first show that $x_{R} \neq(0, \ldots, 0)$. If $R=N$, then the assertion is obvious. If $R>N$, then $[R \leqslant N]=0$, so $m_{R}<n_{R}=\bigoplus_{i} x_{R}^{i}$, which implies that $x_{R} \neq(0, \ldots, 0)$.

We next show that

$$
n_{R}+[R \leqslant N] \leqslant \sum_{i \in \Omega} x_{R}^{i}
$$

Since $n_{R} \oplus[R \leqslant N]=\bigoplus_{i} x_{R}^{i} \leqslant \sum_{i} x_{R}^{i}$, if $n_{R}+[R \leqslant N]=n_{R} \oplus[R \leqslant N]$, then (2.11) holds. Suppose that $n_{R}+[R \leqslant N] \neq n_{R} \oplus[R \leqslant N]$. Then $[R \leqslant N]=1$ and $n_{R} \oplus[R \leqslant N]=0$. Hence $\oplus_{i} x_{R}^{i}=0$. Since $x_{R} \neq(0, \ldots, 0)$, it follows that $\sum x_{R}^{i} \geqslant \beta_{R}=n_{R}+[R \leqslant N]$.

We now construct $u$ satisfying $(\mathrm{C} 1)-(\mathrm{C} 4)$. Since $[R \leqslant M],[R \leqslant N] \in\{0,1\}$ and $m_{R}<n_{R}$, we see that

$$
m_{R} \oplus[R \leqslant M]=m_{R}+[R \leqslant M] \leqslant n_{R}+[R \leqslant N] \leqslant \sum_{i \in \Omega} x_{R}^{i}
$$

By Lemma 15, there exists $u \in \llbracket \beta_{R} \rrbracket^{k}$ satisfying (C1), (C2), and

$$
\operatorname{wt}\left(x_{R}-u\right) \leqslant \min \left\{\beta_{R}-1, k\right\} .
$$

We divide the proof into two cases. 
Case $\mathbf{i}(\boldsymbol{R}>\mathbf{0})$. Since $\min \left\{\beta_{R}-1, k\right\} \leqslant w$, we see that $u$ satisfies (C4). If $u \neq x_{R}$, then $u^{0}<x_{R}^{0}$, so $u$ also satisfies (C3). Suppose that $u=x_{R}$. Then

$$
m_{R} \oplus[R \leqslant M]=\bigoplus_{i \in \Omega} u^{i}=\bigoplus_{i \in \Omega} x_{R}^{i}=n_{R} \oplus[R \leqslant N] .
$$

It follows that $[R \leqslant M] \neq[R \leqslant N]$ since $m_{R}<n_{R}$. Suppose that $[R \leqslant M]=1$ and $[R \leqslant N]=0$. Then $R \leqslant M$ and $R>N$, so $N<R=M$ since $M \leqslant R$. Hence $u$ satisfies (C3). Suppose that $[R \leqslant M]=0$ and $[R \leqslant N]=1$. By (2.12),

$$
m_{R}=\bigoplus_{i \in \Omega} x_{R}^{i}=n_{R} \oplus 1<n_{R}
$$

This implies that $\bigoplus_{i} x_{R}^{i}=0$, and hence that $\sum_{i} x_{R}^{i} \geqslant \beta_{R}$ since $x_{R} \neq(0, \ldots, 0)$. We can now find $\left(\tilde{u}^{0}, \ldots, \tilde{u}^{k-1}\right)$ satisfying $\sum_{i} \tilde{u}^{i}=\sum_{i} x_{R}^{i}-\beta_{R}$ and (C1)-(C3) in the same way as in the proof of Lemma 15. Let $\tilde{u}=\left(\tilde{u}^{0}, \ldots, \tilde{u}^{k-1}\right)$. Since $R>0$,

$$
\operatorname{wt}\left(x_{R}-\tilde{u}\right) \leqslant \min \left\{\beta_{R}, k\right\} \leqslant w,
$$

so $\tilde{u}$ also satisfies $(\mathrm{C} 4)$.

Case ii $(\boldsymbol{R}=\mathbf{0})$. We see that $x_{0} \neq u$ because

$$
\bigoplus_{i \in \Omega} x_{0}^{i}=n_{0} \oplus[0 \leqslant N]=n_{0} \oplus 1
$$

and

$$
\bigoplus_{i \in \Omega} u^{i}=m_{0} \oplus[0 \leqslant M]=m_{0} \oplus 1 .
$$

Thus $u$ satisfies $(\mathrm{C} 3)$. Since $\operatorname{wt}\left(x_{0}-u\right) \leqslant \min \left\{\beta_{0}-1, k\right\}$, we see that (C4) holds when $\min \left\{\beta_{0}-1, k-\left[\beta_{0}<2 k\right]\right\}=\beta_{0}-1$ or $k$. Suppose that

$$
\min \left\{\beta_{0}-1, k-\left[\beta_{0}<2 k\right]\right\}=k-1<\beta_{0}-1 .
$$

Then $k<\beta_{0}<2 k$ and $k-1 \leqslant w$. We show that there exists $\tilde{u}$ satisfying $(\mathrm{C} 1)-(\mathrm{C} 4)$. If $x_{0}^{k-1}=0$, then $u^{k-1}=0$, so $u$ itself satisfies $(\mathrm{C} 1)-(\mathrm{C} 4)$. Suppose that $x_{0}^{k-1} \geqslant 1$. We show that

$$
x_{0}^{0}+\cdots+x_{0}^{k-2} \geqslant m_{0} \oplus 1 \ominus x_{0}^{k-1} .
$$

If $x_{0}^{k-1}=1$, then $n_{0}=\left(\oplus_{i} x_{0}^{i}\right) \ominus 1=x_{0}^{0} \oplus \cdots \oplus x_{0}^{k-2}$, and hence

$$
x_{0}^{0}+\cdots+x_{0}^{k-2} \geqslant x_{0}^{0} \oplus \cdots \oplus x_{0}^{k-2}=n_{0}>m_{0}=m_{0} \oplus 1 \ominus x_{0}^{k-1} .
$$

If $x_{0}^{k-1} \geqslant 2$, then, since $x_{0}^{0} \geqslant x_{0}^{1} \geqslant \cdots \geqslant x_{0}^{k-1} \geqslant 2$ and $2 k>\beta_{0}$,

$$
x_{0}^{0}+\cdots+x_{0}^{k-2} \geqslant 2(k-1) \geqslant \beta_{0}-1 \geqslant m_{0} \oplus 1 \ominus x_{0}^{k-1} .
$$

Thus (2.13) holds. By Lemma 15, there exists $\left(\tilde{u}^{0}, \ldots, \tilde{u}^{k-2}\right)$ such that $\tilde{u}^{0} \oplus \cdots \oplus \tilde{u}^{k-2}=$ $m_{0} \oplus 1 \ominus x_{0}^{k-1}$ and $0 \leqslant x_{0}^{i}-\tilde{u}^{i} \leqslant x_{0}^{0}-\tilde{u}^{0}$. Thus $\left(\tilde{u}^{0}, \ldots, \tilde{u}^{k-2}, x_{0}^{k-1}\right)$ satisfies $(\mathrm{C} 1)-(\mathrm{C} 4)$. This completes the proof. 


\section{References}

[1] M. Albert, R. Nowakowski, and D. Wolfe. Lessons in play: an introduction to combinatorial game theory. CRC Press, Boca Raton, FL, 2nd edition, 2019.

[2] E. R. Berlekamp, J. H. Conway, and R. K. Guy. Winning ways for your mathematical plays. CRC Press, Boca Raton, FL, 2nd edition, 2001.

[3] U. Blass, A. S. Fraenkel, and R. Guelman. How far can Nim in disguise be stretched? J. Combin. Theory Ser. A, 84(2):145-156, 1998.

[4] C. L. Bouton. Nim, a game with a complete mathematical theory. Ann. of Math., 3(1/4):35-39, 1901.

[5] J. H. Conway. On numbers and games. A K Peters, Natick, MA, 2nd edition, 2001.

[6] A. S. Fraenkel and M. Lorberbom. Nimhoff games. J. Combin. Theory Ser. A, 58(1):1-25, 1991.

[7] P. M. Grundy. Mathematics and games. Eureka, 2:6-8, 1939.

[8] P. M. Grundy and C. A. B. Smith. Disjunctive games with the last player losing. Math. Proc. Cambridge Philos. Soc., 52(3):527-533, 1956.

[9] Y. Irie. p-Saturations of Welter's game and the irreducible representations of symmetric groups. J. Algebraic Combin., 48:247-287, 2018.

[10] Y. Irie. Mixed-radix Nim. arXiv:1801.00616, 2018.

[11] J. Kahane and A. J. Ryba. The hexad game. Electron. J. Combin., 8(2) \#R11, 2001.

[12] T. E. Plambeck. Taming the wild in impartial combinatorial games. Integers, 5(1): \#G05, 2005.

[13] T. E. Plambeck and A. N. Siegel. Misère quotients for impartial games. J. Combin. Theory Ser. A, 115(4):593-622, 2008.

[14] A. N. Siegel. Combinatorial game theory, American Mathematical Society, Providence, RI, 2013.

[15] R. P. Sprague. Über mathematische Kampfspiele. Tohoku Math. J., 41:438-444, 1935.

[16] C. P. Welter. The theory of a class of games on a sequence of squares, in terms of the advancing operation in a special group. Indag. Math., 57:194-200, 1954. 